\title{
EDAD Y RECUENTO LEUCOCITARIO COMO FACTORES PRONÓSTICOS EN LEUCEMIA LINFOBLÁSTICA AGUDA: COHORTE HGMLA07
}

\section{AGE AND LEUKOCYTE COUNT AS PROGNOSTIC FACTORS ON ACUTE LYMPHOBLASTIC LEUKEMIA: HGMLALO7 COHORT}

\section{TÍTULO CORTO: EDAD Y RECUENTO LEUCOCITARIO COMO FACTORES PRONÓSTICOS EN LEUCEMIA}

Washington Ladines-Castro ${ }^{1}$, Adrián Santoyo-Sánchez ${ }^{2}$, Etta Rozen-Fuller ${ }^{3}$, Irma Olarte-Carrillo ${ }^{4}$, Adolfo Martínez-Tovar ${ }^{5}$, Humberto Castellanos-Sinco ${ }^{6}$, Juan Collazo-Jaloma ${ }^{7}$, Christian Omar Ramos-Peñafiel ${ }^{8}$

Recibido en octubre 26 de 2015

Aceptado en abril 11 de 2016

\section{RESUMEN}

Con el objetivo de establecer la cifra de corte de leucocitos y edad con implicación pronostica en adultos con leucemia linfoblástica aguda (LLA), se efectuó un estudio observacional, descriptivo y analítico anidado en la cohorte retrospectiva de pacientes con LLA tratados mediante el protocolo institucional HGMLAL07 durante 2007-2014. Se estudiaron 255 pacientes, el 52.9\% $(\mathrm{n}=135)$ correspondieron al género femenino y $47.1 \%(\mathrm{n}=120)$ al género masculino. La media de edad fue de 31 (16-80) años. La supervivencia libre de la enfermedad (SLE) disminuyó en ambos géneros a partir de los 20 años $(\mathrm{p}=0.001)$. La media de leucocitos fue $56.1 \times 10^{9} / \mathrm{L}\left(0.1-850 \times 10^{9} / \mathrm{L}\right)$. La SLE disminuyó significativamente a partir de una cifra igual o mayor de $20 \times 10^{9} / \mathrm{L}(\mathrm{p}<0.05)$. Con esto se puede concluir que emplear puntos de corte para leucocitos y edad obtenidos en poblaciones distintas pudiera condicionar una mala clasificación pronostica y un consiguiente tratamiento subóptimo.

1. Médico Cirujano, Residente de Hematología de tercer año. Servicio de Hematología, Hospital General de México “Dr. Eduardo Liceaga”. Ciudad de México, México. Correo: javi_1411@hotmail.com

2. Médico Pasante de Servicio Social. Unidad de Medicina Experimental, Universidad Nacional Autónoma de México. Ciudad de México, México.Correo: adr_blue_red@ hotmail.com

3. Médico especialista en Hematología. Servicio de Hematología, Hospital General de México “Dr. Eduardo Liceaga”. Ciudad de México, México. Correo: etta.rozen@ gmail.com

142 4. Doctor en Ciencias Biológicas y de la Salud. Laboratorio de Hematología, Hospital General de México "Dr. Eduardo Liceaga”. Ciudad de México, México. Correo: irmaolartec@yahoo.com

5. Doctor en Genética y Biología Molecular. Laboratorio de Hematología, Hospital General de México "Dr. Eduardo Liceaga”. Ciudad de México, México. Correo: mtadolfo73@hotmail.com

6. Médico especialista en Hematología. Servicio de Hematología, Hospital General de México “Dr. Eduardo Liceaga”. Ciudad de México, México. Correo: humbertosinco@ hotmail.com

7. Jefe de Servicio, Médico especialista en Hematología. Servicio de Hematología, Hospital General de México "Dr. Eduardo Liceaga”. Ciudad de México, México. Correo: jcollazo@hotmail.com

8. Jefe del área clínica. Médico especialista en Hematología. Servicio de Hematología, Hospital General de México "Dr. Eduardo Liceaga”. Ciudad de México - México / Departamento de Hematología, Hospital de Alta Especialidad Bicentenario del Instituto de Seguridad y Servicios Sociales de los Trabajadores del Estado. Estado de México, México. Correo: leukemiaCHOP@hotmail.com 
Palabras clave: Edad de Inicio; Leucemia-Linfoma Linfoblástica de Células Precursoras; Pronóstico; Recuento de Leucocitos; Supervivencia sin Enfermedad

\section{ABstract}

In order to establish the cutoff with prognostic implications for white blood cell count and age at diagnosis in adults with acute lymphoblastic leukemia (ALL), we conducted an observational, descriptive and analytical study nested in a retrospective cohort of patients with ALL treated by institutional protocol HGMLAL07 during 2007-2014. We study 255 patients, the $52.9 \%(n=135)$ were female and $47.1 \%(n=120)$ were male. The mean age was $31(16-80)$ years-old. The disease-free survival (DFS) decreases in both genders after 20 years-old $(p=0.001)$. Leukocyte count average was $56.1 \times 10^{9} / \mathrm{L}\left(0.1-850 \times 10^{9} / \mathrm{L}\right)$. DFS decreases significantly from an equal or greater leukocyte count of $20 \times 10^{9} / \mathrm{L}(\mathrm{p}<0.05)$. With this results, we can conclude that use foreign cutoff for age and leukocyte count could determine a bad prognosis stratification and a consequent suboptimal treatment.

Keywords: Age of Onset; Disease-Free Survival; Leukocyte Count; Precursor Cell Lymphoblastic LeukemiaLymphoma; Prognosis

\section{INTRODUCCIÓN}

$\mathrm{L}^{\mathrm{a}}$ a leucemia linfoblástica aguda (LLA) es una neoplasia linfoproliferativa caracterizada por una proliferación descontrolada de precursores linfoides B o T con la consecuente alteración del resto de las líneas hematopoyéticas ${ }^{1,2}$. Diversos grupos en el mundo han diseñado combinaciones secuenciales de quimioterapia como tratamiento ${ }^{3}$, los más novedosos incorporan inhibidores de cinasa de tirosina (Imatinib, Dasatinib, Ponatinib) para portadores de LLA con expresión del cromosoma Philadelphia $(\mathrm{Ph}+)^{4,5}$, así como terapias basadas en anticuerpos monoclonales, siendo el Rituximab (anti-CD20) el más utilizado ${ }^{6}$, otros anticuerpos como Ofatumumab, Epratuzumab , Alemtuzumab y más reciente Blinatumomab brindan resultados esperanzadores para aquellos refractarios a múltiples líneas de quimioterapia o en recaídas de la enfermedad?

Es justamente de los resultados preliminares y finales de los ensayos clínicos ejecutados para el desarrollo de estos medicamentos que se acaban de mencionar, que surge la observación sobre el impacto de ciertos factores sobre la supervivencia libre de la enfermedad y general, obligando a la revisión de los criterios empleados clásicamente para establecer el tipo de riesgo que poseen los pacientes con LLA ${ }^{8,9}$.
La clasificación de riesgo considera marcadores biológicos como lo son la expresión de marcadores de superficie 0 alteraciones citogenéticas específicas $[\mathrm{t}(4 ; 11) /$ ALL1AF4, t $(1 ; 19) /$ PBX-E2A, t $(9 ; 22) / B C R-A B L 1, t(1 ; 19) /$ E2A-PBX] en conjunto con la respuesta temprana al tratamiento o la infiltración al sistema nervioso central ${ }^{10}$. Esta estrategia de clasificación se adaptó originalmente de los grupos pediátricos, considerándose inicialmente dos grupos; el riesgo habitual y el riesgo alto. A pesar de todos los avances, la edad continúa considerándose uno de los principales factores pronósticos para el riesgo de recaída, seguido de la cuenta de leucocitos al diagnóstico. El protocolo MRC UKALL/ECOG E2993 el cual consideró más de 1,500 pacientes identificó 4 factores independientes que influyeron sobre la supervivencia libre de enfermedad: citogenética, inmunofenotipo, edad y cuenta de leucocitos al diagnóstico, de esta última en específico, $30 \times 10^{9} / \mathrm{L}$ para el linaje B y de $100 \times 10^{9} / \mathrm{L}$ para el T son los puntos de corte con implicación pronóstica ${ }^{11}$. Dicha cifra de leucocitos también se ha validado en otras poblaciones que han utilizado el esquema MRC UKALL/ ECOG E2993 ${ }^{12}$. Larson et $\mathrm{al}^{13}$ consideraron esta cifra de leucocitos para determinar los grupos de riesgo para los protocolos terapéuticos del Cancer and Leukemia Group $B$ (CALGB) extendiéndolo a la mayoría de sus protocolos. La edad, continua siendo uno de los principales factores 
pronósticos ya que diversos grupos como el GIMEMA ALL 0288 consideran la edad de corte entre los 30 a 40 años como factor pronóstico ${ }^{14}$. En un primer análisis del protocolo institucional HGMLAL07, la cifra de leucocitos de $30 \times 10^{9} / \mathrm{L}$ no tuvo un impacto determinante sobre la supervivencia, aunque esta si se vio reducida de forma significativa por otros factores como el tipo de riesgo y la expresión del oncogén BCR-ABL1 ${ }^{15}$.

El objetivo principal de este estudio fue establecer la cifra de corte de leucocitos y edad que puedan tener una implicación pronostica en pacientes adultos portadores de leucemia linfoblástica aguda.

\section{MATERIALES Y MÉTODOS}

\section{Diseño del estudio}

Estudio observacional, descriptivo y analítico anidado en una cohorte retrospectiva obtenida de los registros médicos de pacientes con LLA tratados mediante el protocolo institucional HGMLAL07 entre el periodo 20072014 en el Servicio de Hematología del Hospital General de México, y en el Departamento de Hematología del Hospital de Alta Especialidad Bicentenario del ISSSTE (Instituto de Seguridad y Servicios Sociales de los Trabajadores del Estado) durante el periodo 2014-2015.

\section{Pacientes}

Se estudiaron pacientes mayores de 18 años, portadores de leucemia linfoblástica aguda de novo diagnosticados mediante morfología e inmunofenotipo. La cuenta de leucocitos utilizada para el análisis fue la del momento del diagnóstico.

\section{Análisis estadístico}

Se utilizó el software IBM SPSS Statistics versión 22.0 para Windows (Armonk: New York, IBM Corp), para la prueba de contraste de hipótesis se utilizó el test ji-cuadrado, considerándose significativo a un valor de $\mathrm{p} \leq 0.05,95 \%$ IC. Para el análisis de supervivencia se utilizó el método de Kaplan-Meier, la significancia se estableció mediante el test log-rank.

\section{Declaración de consideraciones éticas}

El acceso y uso de los registros médicos fue apegado a la normativa ética estipulada en la declaración de Helsinki de 1975, así como de las consideraciones emitidas por la Secretaria de Salud, vigentes para el territorio mexicano. El protocolo de cohorte retrospectiva fue revisado y aprobados por el Comité de Ética e Investigación del Hospital General de México, bajo el registro: DI/14/103/03/073.

\section{RESULTADOS}

Se estudió un total de 255 pacientes, el $52.9 \%(\mathrm{n}=135)$ correspondieron al género femenino y el $47.1 \%(n=120)$ al género masculino. En cuanto al tipo de riesgo el 37.3\% $(n=95)$ correspondieron a riesgo habitual y el $62.7 \%$ $(n=160)$ a riesgo alto. El 21.2\% $(n=54)$ expresaron el marcador $\mathrm{CD} 20+$ al momento del diagnóstico, la infiltración a sistema nervioso central se reportó en un $1.6 \%(n=4)$ y la expresión del oncogén BCRABL1 solo se presentó en $1.6 \%(n=4)$ de los casos.

Edad y su asociación con la supervivencia: la media de edad fue de 31 años (rango de 16-80 años), acorde a la distribución de edad en su mayoría ( $\mathrm{n}=174,68.2 \%)$ eran menores de 35 años. Los hombres afectados eran más jóvenes con una edad media de 29.4 años (rango 17-66 años), que las mujeres con 34.4 años en promedio (rango 16-80 años). Si existió una diferencia significativa en cuanto a la edad de presentación entre los géneros ( $p=0.001,95 \%$ IC, 34.42- 29.42). Al realizar una comparación en cuanto a la supervivencia acorde al género tanto en el género masculino como al femenino la supervivencia se modificó a partir de los 20 años de edad. Las diferencias de supervivencia tanto por edad como el contraste de edad y género se describen en la Tabla 1 y 2 .

Tabla 1. Supervivencia general a 1 y 5 años acorde edad al diagnóstico.

\begin{tabular}{|c|c|c|}
\hline $\begin{array}{c}\text { Edad } \\
\text { (años) }\end{array}$ & $\begin{array}{c}\text { Supervivencia } \\
\text { General a 5 años (p) }\end{array}$ & $\begin{array}{c}\text { Supervivencia } \\
\text { General a 1 año (p) }\end{array}$ \\
\hline 18 & 0.007 & 0.036 \\
\hline 20 & 0.043 & 0.249 \\
\hline 22 & 0.678 & 0.071 \\
\hline 25 & 0.331 & 0.401 \\
\hline 28 & 0.169 & 0.224 \\
\hline 35 & 0.186 & 0.236 \\
\hline
\end{tabular}


Washington Ladines-Castro, Adrián Santoyo-Sánchez, Etta Rozen-Fuller, Irma Olarte-Carrillo, Adolfo Martínez-Tovar, Humberto Castellanos-Sinco, Juan Collazo-Jaloma, Christian Omar Ramos-Peñafiel

Tabla 2. Supervivencia libre de la enfermedad a 1 y 5 años acorde edad al diagnóstico.

\begin{tabular}{|c|c|c|c|c|}
\hline $\begin{array}{c}\text { Edad } \\
\text { (años) }\end{array}$ & $\begin{array}{c}\text { Supervivencia Libre } \\
\text { de la Enfermedad a 5 } \\
\text { años (p) }\end{array}$ & $\begin{array}{c}\text { Supervivencia Libre } \\
\text { de la Enfermedad a 1 } \\
\text { año (p) }\end{array}$ & $\begin{array}{c}\text { Supervivencia Libre } \\
\text { de la Enfermedad a 5 } \\
\text { años (p) }\end{array}$ & $\begin{array}{c}\text { Supervivencia Libre } \\
\text { de la Enfermedad a 1 } \\
\text { año (p) }\end{array}$ \\
\hline 18 & 0.015 & 0.057 & 0.015 & 0.057 \\
\hline 20 & $0.001^{* *}$ & $0.003^{* *}$ & $0.001^{* *}$ & $0.003^{* *}$ \\
\hline 22 & 0.196 & 0.080 & 0.889 & 0.080 \\
\hline 25 & 0.889 & 0.941 & 0.689 & 0.941 \\
\hline 28 & 0.426 & 0.605 & 0.426 & 0.605 \\
\hline 35 & 0.097 & 0.163 & 0.097 & 0.163 \\
\hline 40 & 0.260 & 0.481 & 0.260 & 0.481 \\
\hline
\end{tabular}

Leucocitos y su asociación con la supervivencia: se obtuvo una media de leucocitos de $56.1 \times 10^{\%} / \mathrm{L}$ (rango 0.1- 850 x $\left.10^{9} / \mathrm{L}\right)$, un poco mayor para el género femenino $(60.2 \mathrm{x}$ $\left.10^{9} / \mathrm{L}\right)$ al compararse con el género masculino $\left(52.5 \times 10^{9} / \mathrm{L}\right)$.
No existieron diferencias estadísticamente significativas acorde a la cifra de leucocitos y género $(\mathrm{p}=0.558,95 \%$ IC; 60.21 vs 52.56 ). Los resultados de la supervivencia acorde a los diferentes rangos de leucocitos se describen en la Tabla 3.

Tabla 3. Supervivencia libre de la enfermedad a 1 y 5 años según cifra de leucocitos al diagnóstico.

\begin{tabular}{|c|c|c|c|c|}
\hline $\begin{array}{c}\text { Cifra de leucocitos } \\
(\mathrm{x} \mathrm{10} / \mathrm{L})\end{array}$ & $\begin{array}{c}\text { Supervivencia } \\
\text { general a 5 años (p) }\end{array}$ & $\begin{array}{c}\text { Supervivencia } \\
\text { general a 1 año (p) }\end{array}$ & $\begin{array}{c}\text { Supervivencia Libre } \\
\text { de la Enfermedad a 5 5 } \\
\text { años (p) }\end{array}$ & $\begin{array}{c}\text { Supervivencia Libre } \\
\text { de la Enfermedad a 1 } \\
\text { año (p) }\end{array}$ \\
\hline 20 & 0.068 & 0.207 & 0.003 & 0.009 \\
\hline 22 & 0.036 & 0.158 & 0.005 & 0.014 \\
\hline 25 & 0.041 & 0.120 & 0.005 & 0.008 \\
\hline 28 & 0.033 & 0.093 & 0.005 & 0.005 \\
\hline 30 & 0.005 & 0.026 & 0.003 & 0.006 \\
\hline 40 & 0.014 & 0.057 & 0.003 & 0.007 \\
\hline 50 & 0.007 & 0.007 & 0.001 & 0.002 \\
\hline 60 & 0.000 & 0.000 & 0.000 & 0.000 \\
\hline 70 & 0.000 & 0.002 & 0.000 & 0.000 \\
\hline 80 & 0.000 & 0.001 & 0.000 & 0.001 \\
\hline 90 & 0.000 & 0.001 & 0.000 & 0.001 \\
\hline
\end{tabular}

Supervivencia: un $27 \%$ de los pacientes permanecían vivos tras un seguimiento de 5 años. La gráfica de supervivencia global a 5 años y al año de seguimiento acorde a la edad se describe en la Figura 1 y 2 . La supervivencia libre de enfermedad acorde a la cifra de leucocitos de $20 \times 10^{9} / \mathrm{L}$ se describe en la Figura 3. 
Figura 1. Supervivencia global a 5 años según grupo de edad.

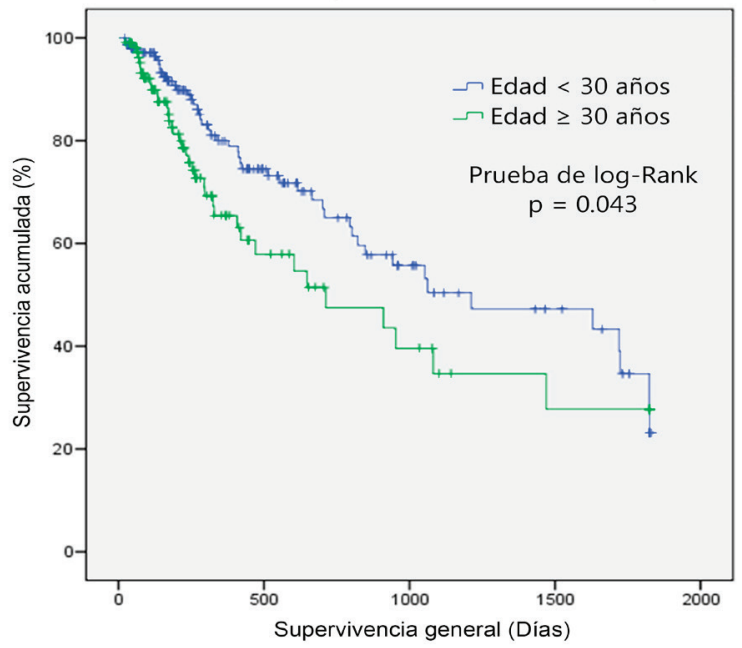

Figura 3. Supervivencia libre de la enfermedad a 5 años acorde a la cifra de leucocitos.

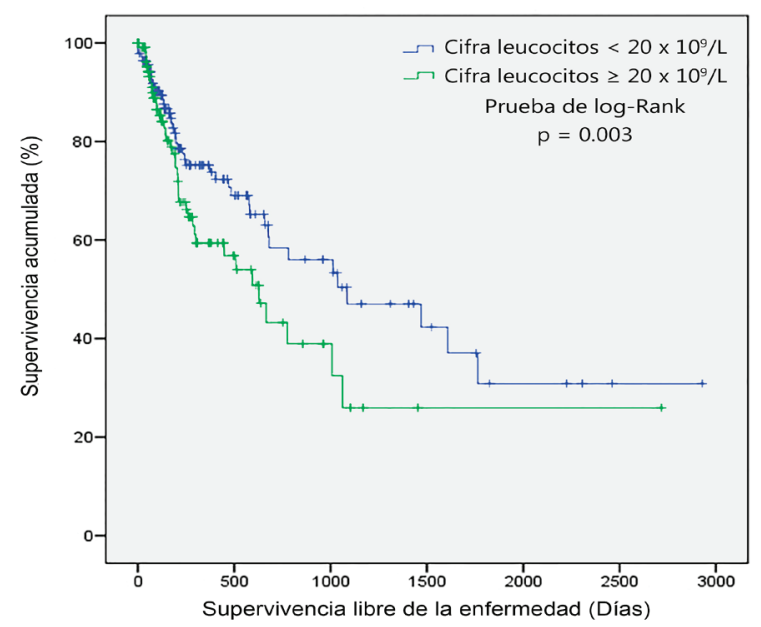

DISCUSIÓN

Actualmente el médico hematólogo dispone de un número cada vez mayor de estudios avanzados de biología molecular para clasificar, elegir o modificar tratamientos y establecer el pronóstico de los pacientes con leucemia ${ }^{16,17}$. Sin embargo, dos de los cuatro factores que realmente impactan sobre la supervivencia libre de la enfermedad, la edad y la cifra de leucocitos al momento del diagnóstico, son variables clínicas de fácil detección ${ }^{14,18}$. La media de edad obtenida en la población analizada es similar a las reportadas en Latinoamérica, y ligeramente menor a la de estudios anglosajones ${ }^{19-22}$. El género a pesar de influir
Figura 2. Supervivencia global a 1 año según grupo de edad.

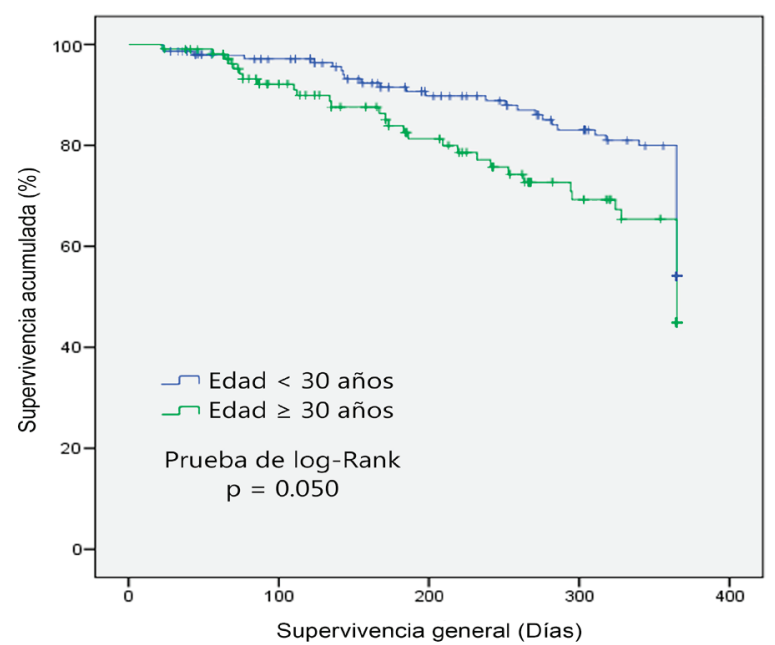

significativamente en la edad al momento de diagnóstico, menor para el género masculino, no fue un factor de impacto para la supervivencia libre de la enfermedad, ensombreciendo el pronóstico tanto para hombres como mujeres con edad igual o mayor a los 20 años.

Respecto al número de leucocitos en sangre periférica al momento del diagnóstico una cifra igual o mayor a $20 \times 10^{9} / \mathrm{L}$ fue a partir de la cual se impactó de forma significativa sobre la disminución de la supervivencia libre de la enfermedad a uno y cinco años, menor a las cifras típicamente empleadas para estratificar el riesgo por las grandes series de pacientes con leucemia. En Alemania un primer estudio multicéntrico prospectivo que incluyó 368 pacientes en la década de los ochenta estableció como punto de corte pronóstico para leucocitos $30 \times 10^{9} / \mathrm{L}^{22}$, cifra reciente empleada y ratificada por el GMALL (German Multicenter Study Group for Adult ALL) que empleo datos de 1,648 pacientes $^{23}$. El protocolo MRC UKALL/ECOG E2993 en Reino Unido también utilizó esa cifra de corte, así como el estudio de Wang et al en China empleando el mismo esquema ${ }^{11,12}$. Por su parte en Italia, las vertientes de la gran cohorte GIMEMA (Gruppo Italiano Malattie Ematologiche dell'Adulto) obtuvieron una media de $13.6 \times 10^{9} / \mathrm{L}$ y estratifican a sus pacientes en 3 grupos: cifras de leucocitos menores a $50 \times 10^{\circ} / \mathrm{L}, 74 \%$ de los pacientes se ubican en este rango con riesgo habitual; $12 \%$ presentan cifras entre $50-100 \times 10^{9} / \mathrm{L}$; y un $14 \%$ con recuento mayor a $100 \times 10^{9} / \mathrm{L}$ considerándose de muy alto riesgo, ${ }^{9,14}$. El MD Anderson Cancer Center, referente mundial del tratamiento de leucemia con el Hyper-CVAD, identificó que cifras de leucocitos superiores a 50 × $10^{9} / \mathrm{L}$ 
otorgaban un pronóstico adverso, similar a los hallazgos de los trabajos con población pediátrica ${ }^{8}$.

Conjuntando las dos variables de mayor impacto en este trabajo, es conveniente recordar la teoría de la inmunosenescencia, la cual sugiere que tanto la función como el número de células hematopoyéticas disminuyen con la edad ${ }^{24}$, por lo cual a pesar de no encontrar grandes diferencias de edad entre la presente serie y los estudios previamente citados, lo esperado sería encontrar cifras discretamente mayores de leucocitos, sin embargo ocurre lo contrario, por lo cual seguramente existen más factores (clínicas, hematológicas y genéticas) detrás de este hallazgo.

Esto finalmente invita a la reflexión sobre el diseño de los esquemas de tratamiento ya que un porcentaje nada despreciable de pacientes debuta con hiperleucocitosis que, si bien no alcanza los puntos de corte típicos, si sobrepasan los $20 \times 10^{9} / \mathrm{L}$, con la consecuente implicación pronostica que obligaría a intensificar el tratamiento y adicionar estrategias de profilaxis a sistema nervioso central.

Utilizar un punto de corte universal para estratificar el riesgo de los pacientes con leucemia es válido cuando los pacientes comparten las características (particularmente genéticas) de la población del estudio referente. De no ser así, se pudieran estar condicionando dosis subóptimas de quimioterapia, con el consiguiente fracaso terapéutico. Con base a los datos mostrados esto parece estar ocurriendo en la población mexicana, y seguramente en toda Latinoamérica, por lo que se recomienda reducir el punto de corte para leucocitos al momento del diagnóstico.

\section{DECLARACIÓN SOBRE CONFLICTO DE INTERESES}

Los autores manifiestan que durante la ejecución del trabajo o la redacción del manuscrito, no han incidido intereses, recibido valores monetarios, ni presentan relación laboral o de alguna otra especie con empresas privadas, agrupaciones civiles o instituciones financiadoras.

\section{REFERENCIAS BIBLOGRÁFICAS}

1. Swerdlow SH, Campo E, Harris NL, Jaffe ES, Pileri SA, Stein $\mathrm{H}$, et al. editors. WHO Classification of Tumours of Haematopoietic and Lymphoid Tissues. 4th ed. World Health Organization Classification of Tumours of Haematopoietic and Lymphoid Tissue. Lyon: International Agency for Research on Cancer Press; 2008.
2. Nath SV, Nicholson I, Tapp H, Zola H, Zannettino ACW, Revesz T. Reticulin fibres anchor leukaemic blasts in the marrow of patients with acute lymphoblastic leukaemia. Med Hypotheses. 2011;77(3):333-5.

3. Gökbuget N, Hoelzer D. Treatment of adult acute lymphoblastic leukemia. Semin Hematol. 2009;46(1):64-75.

4. Talpaz M, Shah NP, Kantarjian H, Donato N, Nicoll $\mathrm{J}$, Paquette R, et al. Dasatinib in imatinib-resistant Philadelphia chromosome-positive leukemias. N Engl J Med. 2006;354(24):2531-41.

5. Sanford DS, Kantarjian H, O’Brien S, Jabbour E, Cortes J, Ravandi F. The role of ponatinib in Philadelphia chromosome-positive acute lymphoblastic leukemia. Expert Rev Anticancer Ther. 2015;15(4):365-73.

6. Portell CA, Advani AS. Antibody therapy for acute lymphoblastic leukemia. Curr Hematol Malig Rep. 2012;7(2):153-9.

7. Ai J, Advani A. Current status of antibody therapy in ALL. Br J Haematol. 2015;168(4):471-80.

8. Pui CH, Carroll WL, Meshinchi S, Arceci RJ. Biology, risk stratification, and therapy of pediatric acute leukemias: an update. J Clin Oncol. 2011;29(5):551-65.

9. Chiaretti S, Vitale A, Cazzaniga G, Orlando SM, Silvestri D, Fazi P, et al. Clinico-biological features of 5202 patients with acute lymphoblastic leukemia enrolled in the Italian AIEOP and GIMEMA protocols and stratified in age cohorts. Haematologica. 2013;98(11):1702-10.

10. Hoelzer D, Thiel E, Löffler H, Ganser A, Heimpel H, Büchner T, et al. Risk groups in adult acute lymphoblastic leukemia. Haematol Blood Transfus. 1987;30:104-10.

11. Rowe JM, Buck G, Burnett AK, Chopra R, Wiernik PH, Richards SM, et al. Induction therapy for adults with acute lymphoblastic leukemia: results of more than 1500 patients from the international ALL trial: MRC UKALL XII/ECOG E2993. Blood. 2005;106(12):3760-7.

12. Wang H, Chen XQ, Geng QR, Liu PP, Lin GN, Xia ZJ, et al. Induction therapy using the MRC UKALLXII/ECOG E2993 protocol in Chinese adults with acute lymphoblastic leukemia. Int J Hematol. 2011;94(2):163-8.

13. Larson RA, Dodge RK, Burns CP, Lee EJ, Stone RM, Schulman $P$, et al. A five-drug remission induction regimen with intensive consolidation for adults with acute lymphoblastic leukemia: cancer and leukemia group B study 8811. Blood. American Society of Hematology; 1995;85(8):2025-37.

14. Annino L, Vegna ML, Camera A, Specchia G, Visani G, Fioritoni G, et al. Treatment of adult acute lymphoblastic leukemia (ALL): long-term follow-up of the GIMEMA ALL 0288 randomized study. Blood. 2002;99(3):863-71.

15. Ramos C, Rozen E, León M, Martínez TA, Olarte I, Catellanos H, et al. [Results of treatment of acute lymphoblastic leukemia in two cohorts of Mexican patients]. Rev Med Chil. 2011;139(9):1135-42. 
16. Jaso J, Thomas DA, Cunningham K, Jorgensen JL, Kantarjian HM, Medeiros LJ, et al. Prognostic significance of immunophenotypic and karyotypic features of Philadelphia positive B-lymphoblastic leukemia in the era of tyrosine kinase inhibitors. Cancer. 2011;117(17):4009-17.

17. Jiménez-Morales S, Miranda-Peralta E, Saldaña-Alvarez Y, Perez-Vera P, Paredes-Aguilera R, Rivera-Luna R, et al. BCR-ABL, ETV6-RUNX1 and E2A-PBX1: prevalence of the most common acute lymphoblastic leukemia fusion genes in Mexican patients. Leuk Res. 2008;32(10):1518-22.

18. Ganzel C, Rowe JM. Prognostic factors in adult acute leukemia. Hematol Oncol Clin North Am. 2011;25(6):1163-87.

19. Dores GM, Devesa SS, Curtis RE, Linet MS, Morton LM. Acute leukemia incidence and patient survival among children and adults in the United States, 2001-2007. Blood. 2012;119(1):34-43.

20. Sant M, Allemani C, Tereanu C, Angelis RD, Capocaccia $\mathrm{R}$, Marcos-gragera $\mathrm{R}$, et al. Incidence of hematologic malignancies in Europe by morphologic subtype: results of the HAEMACARE project. Blood. 2010;116(19):3724-34.
21. De França Azevedo I, Da Silva Júnior RMP, de Vasconcelos AVM, Das Neves WB, De Barros Correia Melo FC, Melo RAM. Frequency of p190 and p210 BCR$\mathrm{ABL}$ rearrangements and survival in Brazilian adult patients with acute lymphoblastic leukemia. Rev Bras Hematol Hemoter. 36(5):351-5.

22. Hoelzer D, Thiel E, Löffler H, Büchner T, Ganser A, Heil $G$, et al. Prognostic factors in a multicenter study for treatment of acute lymphoblastic leukemia in adults. Blood. 1988;71(1):123-31.

23. Gokbuget N, Kneba M, Raff T, Trautmann H, Bartram CR, Arnold R, et al. Adult patients with acute lymphoblastic leukemia and molecular failure display a poor prognosis and are candidates for stem cell transplantation and targeted therapies. Blood. American Society of Hematology. 2012;120(9):1868-76.

24. Van Zant G, Liang Y. Concise Review: Hematopoietic Stem Cell Aging, Life Span, and Transplantation. Stem Cells Transl Med. 2012;1(9):651-7.

Para citar este artículo: Ladines-Castro W, Santoyo-Sánchez A, Rozen-Fuller E, OlarteCarrillo I, Martínez-Tovar A, Castellanos-Sinco H, et al. Edad y recuento leucocitario como factores pronósticos en leucemia linfoblástica aguda: cohorte HGMLA07. Duazary. 2016 julio; 13 (2): 142 - 148 